\title{
GENERALIZED FRACTIONS AND HUGHES' GRADE- THEORETIC ANALOGUE OF THE COUSIN COMPLEX
}

\author{
by R. Y. SHARP and M. YASSI
}

(Received 23 December, 1988)

0. Introduction. Let $A$ be a commutative Noetherian ring (with non-zero identity). The Cousin complex $C(A)$ for $A$ is described in [19, Section 2]: it is a complex of $A$-modules and $A$-homomorphisms

$$
0 \stackrel{d^{-2}}{\longrightarrow} A \stackrel{d^{-1}}{\longrightarrow} A^{0} \stackrel{d^{0}}{\longrightarrow} A^{1} \longrightarrow \ldots \longrightarrow A^{n} \stackrel{d^{n}}{\longrightarrow} A^{n+1} \longrightarrow \ldots
$$

with the property that, for each $n \in \mathbb{N}_{0}$ (we use $\mathbb{N}_{0}$ to denote the set of non-negative integers),

$$
A^{n}=\bigoplus_{\substack{\mathfrak{p} \in \operatorname{Spec}(A) \\ \text { ht } \mathfrak{p}=n}}^{\bigoplus}\left(\text { Coker } d^{n-2}\right)_{\mathfrak{v}} .
$$

Cohen-Macaulay rings can be characterized in terms of the Cousin complex: $A$ is a Cohen-Macaulay ring if and only if $C(A)$ is exact $[19,(4.7)]$. Also, the Cousin complex provides a natural minimal injective resolution for a Gorenstein ring (see $[19,(5.4)]$ ).

Various more general Cousin complexes can be constructed. For an $A$-module $M$, we can construct the Cousin complex $C(M)$ for $M$ as in [19, Section 2]: for that we concentrate attention on prime ideals in $\operatorname{Supp}(M)$, the support of $M$. More generally still, we can, for any filtration $\mathscr{F}[21,1.1]$ of $\operatorname{Spec}(A)$ that admits $M$, construct the Cousin complex $C(\mathscr{F}, M)$ for $M$ with respect to $\mathscr{F}[21,1.3]$.

Recently, K. R. Hughes [10] has introduced a grade-theoretic analogue of the Cousin complex. We describe the construction of this now: it employs Rees's concept of the grade of a proper ideal $\mathfrak{b}$ of $A$. Recall [17] that grade $\mathfrak{b}$ is the length of each maximal $A$-sequence in $\mathfrak{b}$ (that is the length of each $A$-sequence which is maximal with respect to the property of being contained in $\mathfrak{b}$ ). It will be convenient for us to adopt the convention whereby the grade of the improper ideal $A$ of $A$ is regarded as $\infty$. For each $n \in \mathbb{N}_{0}$, set

$$
\mathscr{G}(n)=\{\mathfrak{b}: \mathfrak{b} \text { is an ideal of } A \text { and grade } \mathfrak{b} \geqslant n\} .
$$

Then $\mathscr{G}(n)$ is a directed set under reverse inclusion and we can modify the ideas in $[20,2.2]$ in an obvious way to produce functors

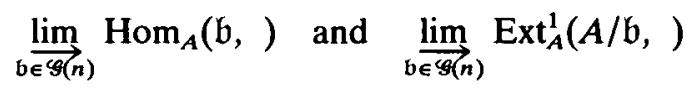

from the category of all $A$-modules and $A$-homomorphisms to itself.

Set $E^{0}=A$. It is also convenient to set $D^{-2}=0$ and $D^{-1}=A$, and to denote the zero homomorphism from 0 to $A$ by $f^{-2}$. Suppose, inductively, that $n \in \mathbb{N}_{0}$ and we have constructed a complex

$$
0 \stackrel{f^{-2}}{\longrightarrow} A \stackrel{f^{-1}}{\longrightarrow} D^{0} \stackrel{f^{0}}{\longrightarrow} D^{1} \longrightarrow \ldots \longrightarrow D^{n-2} \stackrel{f^{n-2}}{\longrightarrow} D^{n-1},
$$

Glasgow Math. J. 32 (1990) 173-188. 
an $A$-module $E^{n}$ and an exact sequence

$$
D^{n-2} \stackrel{f^{n-2}}{\longrightarrow} D^{n-1} \stackrel{\pi_{n}}{\longrightarrow} E^{n} \longrightarrow 0 .
$$

(This is certainly the case for $n=0$ : take $\pi_{0}$ to be the identity mapping of $A$ to itself.)

For each ideal $\mathfrak{b} \in \mathscr{G}(n+1)$, the exact sequence

$$
0 \longrightarrow \mathfrak{b} \longrightarrow A \longrightarrow A / \mathfrak{b} \longrightarrow 0
$$

yields an induced exact sequence

$$
E^{n} \longrightarrow \operatorname{Hom}_{A}\left(\mathfrak{b}, E^{n}\right) \longrightarrow \operatorname{Ext}_{A}^{1}\left(A / \mathfrak{b}, E^{n}\right) \longrightarrow 0 ;
$$

since passage to direct limits preserves exactness, we obtain an exact sequence

We set

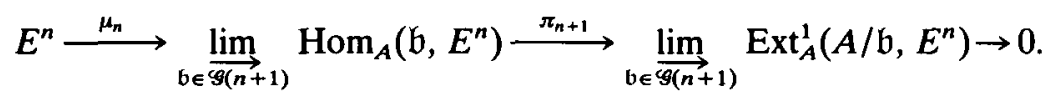

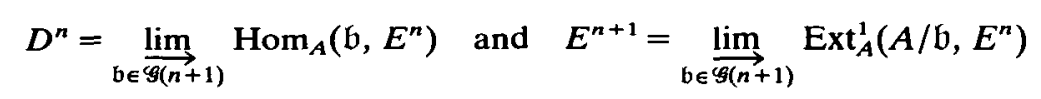

and define $f^{n-1}: D^{n-1} \rightarrow D^{n}$ by $f^{n-1}=\mu_{n} \circ \pi_{n}$. This completes the inductive step; the construction yields a complex

$$
0 \longrightarrow A \stackrel{f^{-1}}{\longrightarrow} D^{0} \stackrel{f^{0}}{\longrightarrow} D^{1} \longrightarrow \ldots \longrightarrow D^{n} \stackrel{f^{n}}{\longrightarrow} D^{n+1} \longrightarrow \ldots
$$

which Hughes calls the 'grade-theoretic analogue of the Cousin complex'.

In addition, Hughes remarks that his construction uses 'generalized modules of fractions at the Gabriel filter generated by $\mathscr{G}(n)$ ' in the sense of J. Lambek [11].

Now the present first author and $\mathrm{H}$. Zakeri introduced a concept of module of generalized fractions in commutative algebra in [23]. Since that paper appeared, there have been several further papers, such as [3], [4], [5], [6], [8], [9], [14], [15], [18], [22], $[24],[25],[26],[27]$ and [28], which have shown that this concept has many interactions with topics of recent and current interest in commutative algebra, especially in commutative Noetherian ring theory. In particular, there are strong links between Cousin complexes and these modules of generalized fractions: it was shown in [18, (3.4)] that, for an $A$-module $M$ such that $\operatorname{Ass}(M)$ has only finitely many minimal members and a filtration $\mathscr{F}$ of $\operatorname{Spec}(A)$ which admits $M$, the Cousin complex $C(\mathscr{F}, M)$ mentioned above is actually isomorphic to a complex of modules of generalized fractions in the sense of [23].

In view of this result, of Hughes' description of his complex as a 'grade-theoretic analogue of the Cousin complex', and of the relevance to his construction of generalized modules of fractions in the sense of Lambek [11], it seems desirable that relationships between his complex and the modules of generalized fractions of Sharp and Zakeri should be explored. It is the purpose of the present paper to do just that.

In Section 1, we shall discuss a sort of 'generalized ideal transform' determined by a system of ideals of $A$ in the sense of [1]: such a system is a non-empty set $\Phi$ of ideals of $A$ with the property that, whenever $\mathfrak{a}, \mathfrak{b} \in \Phi$, then there exists $\mathfrak{c} \in \Phi$ such that $\mathfrak{c} \subseteq \mathfrak{a b}$. It should be observed that the set $\mathscr{G}(n)$ (for $n \in \mathbb{N}_{0}$ ) of all ideals of $A$ of grade at least $n$ is an example of such a system of ideals. The generalized ideal transform determined by $\Phi$ is 
the functor

$$
D_{\Phi}:=\underset{\mathfrak{b} \in \Phi}{\lim } \operatorname{Hom}_{A}(\mathfrak{b},)
$$

from the category of all $A$-modules and $A$-homomorphisms to itself. (Of course, $\Phi$ is a directed set with respect to reverse inclusion.)

We shall use this idea to produce, for an $A$-module $M$ and a family $\left(\Phi_{n}\right)_{n \in \mathbb{N}}$ of systems of ideals of $\boldsymbol{A}$ (here, $\mathbb{N}$ denotes the set of positive integers), a complex

$$
0 \stackrel{h^{-2}}{\longrightarrow} M \stackrel{h^{-1}}{\longrightarrow} K^{0} \stackrel{h^{0}}{\longrightarrow} K^{1} \longrightarrow \ldots \longrightarrow K^{n} \stackrel{h^{n}}{\longrightarrow} K^{n+1} \longrightarrow \ldots
$$

which can be regarded as a generalization of Hughes' complex described above.

In Section 2, we shall review some of the basic properties of the modules of generalized fractions introduced by Sharp and Zakeri in [23], and we shall show how a chain of triangular sets on $A$ in the sense of $[14, p .420]$ gives rise to a family of systems of ideals of $A$, and therefore to associated 'generalized Hughes complexes'.

Section 3 contains the main results of the paper. In it, we compare some of our 'generalized Hughes complexes' with complexes of modules of generalized fractions. We shall show, among other things, that every module of generalized fractions of an $A$-module $M$ is isomorphic to a term in a suitable 'generalized Hughes complex' as described above. We shall also use our results, together with basic facts from the theory of generalized fractions, to recover some of Hughes' results about his grade-theoretic analogue of the Cousin complex.

Throughout the paper, $A$ will denote a commutative Noetherian ring (with non-zero identity). In some of the discussion, the Noetherian hypothesis will not be needed, and so we shall use $R$ to denote, again throughout the paper, a commutative ring (with non-zero identity). Also, $\mathscr{C}(R)$ will denote the category of all $R$-modules and $R$-homomorphisms, and, of course, $\mathscr{C}(A)$ will have a similar meaning for $A$.

\section{Generalized ideal transforms and generalized Hughes complexes.}

1.1 Definitions. A system of ideals of $R[1]$ is a non-empty set $\Phi$ of ideals of $R$ such that, whenever $I, J \in \Phi$, there exists $K \in \Phi$ such that $K \subseteq I J$.

Such a system of ideals $\Phi$ determines the $\Phi$-torsion functor $\Gamma_{\Phi}: \mathscr{C}(R) \rightarrow \mathscr{C}(R)$. This is the subfunctor of the identity functor on $\mathscr{C}(R)$ for which

$$
\Gamma_{\Phi}(M)=\{m \in M: I m=0 \text { for some } I \in \Phi\}
$$

for each $R$-module $M$.

Note that $\Gamma_{\Phi}$ is denoted by $L_{\Phi}$ in [1], and is referred to in that paper as the 'general local cohomology functor with respect to $\Phi$ '. (The underlying rings in [1] are all assumed to be Noetherian, but we are not making such an assumption in this section.)

There are many examples of systems of ideals. One which is particularly relevant to this paper is the set $\mathscr{G}(n)$ (for $n \in \mathbb{N}_{0}$ ) of all ideals of our commutative Noetherian ring $A$ which have grade at least $n$. We shall provide other examples in Section 2. 
1.2 Definition and Notation. Let $\Phi$ be a system of ideals of $R$. Since $\Phi$ is a directed set with respect to reverse inclusion, it is straightforward to adapt the ideas of $[20,2.2]$ in an obvious way to produce functors

$$
D_{\Phi}:=\underset{I \in \Phi}{\lim _{I}} \operatorname{Hom}_{R}(I,) \text { and } H_{\Phi}^{1}:=\underset{I \in \Phi}{\lim _{I}} \operatorname{Ext}_{R}^{1}(R / I,)
$$

from $\mathscr{C}(R)$ to itself. The functor $D_{\Phi}$ is called the generalized ideal transform determined by $\Phi$, or, more briefly, the $\Phi$-transform.

Note also that we can similarly define a functor

$$
\underset{I \in \Phi}{\lim _{I}} \operatorname{Hom}_{R}(R / I,)
$$

from $\mathscr{C}(R)$ to itself, and that this is naturally equivalent to $\Gamma_{\Phi}$.

For each $R$-module $M$ and each $I \in \Phi$, the exact sequence

$$
0 \longrightarrow I \longrightarrow R \rightarrow R / I \longrightarrow 0
$$

induces an exact sequence

$$
0 \longrightarrow \operatorname{Hom}_{R}(R / I, M) \longrightarrow \operatorname{Hom}_{R}(R, M) \rightarrow \operatorname{Hom}_{R}(I, M) \longrightarrow \operatorname{Ext}_{R}^{1}(R / I, M) \longrightarrow 0
$$

(because $R$ is $R$-projective). Since passage to direct limits preserves exactness, it follows that there is an exact sequence

$$
0 \longrightarrow \Gamma_{\Phi}(M) \longrightarrow M \stackrel{\eta_{\Phi}(M)}{\longrightarrow} D_{\Phi}(M) \stackrel{\zeta_{\Phi}(M)}{\longrightarrow} H_{\Phi}^{1}(M) \longrightarrow 0
$$

in $\mathscr{C}(R)$. Furthermore, it is straightforward to check that, as $M$ varies through the category $\mathscr{C}(R)$, the $\eta_{\Phi}(M)$ and $\zeta_{\Phi}(M)$ constitute morphisms of functors

$$
\eta_{\Phi}: \mathrm{Id} \longrightarrow D_{\Phi} \quad \text { and } \quad \xi_{\Phi}: D_{\Phi} \longrightarrow H_{\Phi}^{1}
$$

from $\mathscr{C}(R)$ to itself. (Of course, Id here denotes the identity functor from $\mathscr{C}(R)$ to itself.)

The reader should note the effect of $\eta_{\Phi}(M)$ on an element $x \in M$ : if we let, for $I \in \Phi$,

$$
\theta(\Phi, I)(M): \operatorname{Hom}_{R}(I, M) \longrightarrow D_{\Phi}(M)
$$

be the canonical homomorphism, and $\lambda_{l, x}: I \rightarrow M$ the homomorphism for which $\lambda_{I, x}(r)=r x$ for all $r \in I$, then $\left(\eta_{\Phi}(M)\right)(x)=(\theta(\Phi, I)(M))\left(\lambda_{I, x}\right)$.

We show next how, given a family $\mathscr{S}=\left(\Phi_{i}\right)_{i \in \mathbb{N}}$ of systems of ideals of $R$ and an $R$-module $M$, we can construct a complex

$$
0 \stackrel{h^{-2}}{\longrightarrow} M \stackrel{h^{-1}}{\longrightarrow} K^{0} \stackrel{h^{0}}{\longrightarrow} K^{1} \longrightarrow \ldots \longrightarrow K^{n} \stackrel{h^{n}}{\longrightarrow} K^{n+1} \longrightarrow \ldots
$$

which can be regarded as a generalization of Hughes' complex of [10].

1.3 Construction. Let $\mathscr{S}=\left(\Phi_{i}\right)_{i \in \mathbb{N}}$ be a family of systems of ideals of $R$, and let $M$ be an $R$-module. It will be convenient to write $K^{-2}=0, K^{-1}=M$, and to use $h^{-2}: K^{-2} \rightarrow K^{-1}$ to denote the zero homomorphism. Set $E^{0}=M$, and let $\pi_{0}: K^{-1} \rightarrow E^{0}$ be the identity mapping of $M$ to itself.

Suppose, inductively, that $n \in \mathbb{N}_{0}$ and we have constructed a complex

$$
0 \longrightarrow M \stackrel{h^{-1}}{\longrightarrow} K^{0} \longrightarrow \ldots \longrightarrow K^{n-2} \stackrel{h^{n-2}}{\longrightarrow} K^{n-1},
$$


an $R$-module $E^{n}$ and an epimorphism $\pi_{n}: K^{n-1} \rightarrow E^{n}$ for which the sequence

$$
K^{n-2} \stackrel{h^{n-2}}{\longrightarrow} K^{n-1} \stackrel{\pi_{n}}{\longrightarrow} E^{n} \longrightarrow 0
$$

is exact. This is certainly the case when $n=0$.

To construct the next term and the next homomorphism, we apply the ideas of 1.2 to the system of ideals $\Phi_{n+1}$ and the $R$-module $E^{n}$. We obtain an exact sequence

$$
E^{n} \stackrel{\eta_{\Phi_{n+1}}\left(E^{n}\right)}{\longrightarrow} D_{\Phi_{n+1}}\left(E^{n}\right) \stackrel{\zeta_{\Phi_{n+1}}\left(E^{n}\right)}{\longrightarrow} H_{\Phi_{n+1}}^{1}\left(E^{n}\right) \longrightarrow 0 .
$$

We define $K^{n}:=D_{\Phi_{n+1}}\left(E^{n}\right)$ and $E^{n+1}:=H_{\Phi_{n+1}}^{1}\left(E^{n}\right)$; also, we set

$$
h^{n-1}=\eta_{\Phi_{n+1}}\left(E^{n}\right) \circ \pi_{n}: K^{n-1} \longrightarrow K^{n}
$$

and $\pi_{n+1}=\zeta_{\Phi_{n+1}}\left(E^{n}\right): K^{n} \rightarrow E^{n+1}$. Since

$$
h^{n-1} \circ h^{n-2}=\eta_{\Phi_{n+1}}\left(E^{n}\right) \circ \pi_{n} \circ h^{n-2}=0
$$

by the inductive hypothesis, and we have an exact sequence

$$
K^{n-1} \stackrel{h^{n-1}}{\longrightarrow} K^{n} \stackrel{\pi_{n+1}}{\longrightarrow} E^{n+1} \longrightarrow 0
$$

because $\pi_{n}$ is surjective, the inductive step in the construction is complete.

We shall call the complex

$$
0 \longrightarrow M \stackrel{h^{-1}}{\longrightarrow} K^{0} \stackrel{h^{0}}{\longrightarrow} K^{1} \longrightarrow \ldots \longrightarrow K^{i} \stackrel{h^{i}}{\longrightarrow} K^{i+1} \longrightarrow \ldots
$$

that results from this construction the generalized Hughes complex for $M$ with respect to the family of systems of ideals $\mathscr{Y}$; we shall denote it by $\mathscr{H}(\mathscr{S}, M)$.

1.4 Remark. It should be noted that, if we let $\mathscr{G}$ denote the family of systems of ideals of our commutative Noetherian ring $A$ given by $\mathscr{G}=(\mathscr{G}(n))_{n \in \mathbb{N}}$, where, for each $n \in \mathbb{N}$, the system $\mathscr{G}(n)$ is the set of all ideals of $A$ of grade at least $n$, then the generalized Hughes complex $\mathscr{H}(\mathscr{G}, A)$ for $A$ with respect to $\mathscr{G}$ is just the grade-theoretic analogue of the Cousin complex which Hughes constructed in [10].

In the next section, we shall give more examples of generalized Hughes complexes; these will be defined using families of systems of ideals of $A$ that come from chains of triangular sets on $A$.

2. Some basic results about generalized fractions. In this section, we shall work over our commutative ring $R$. We begin with a brief review of some of the main elements of the theory of modules of generalized fractions.

Let $M$ be an $R$-module. The construction of a module of generalized fractions of $M$ requires a (positive integer $n$ and a) triangular subset (see $[23,2.1]$ ) $U \subseteq R^{n}$; the construction produces a module $U^{-n} M$, called the module of generalized fractions of $M$ with respect to $U$, whose elements, called generalized fractions, have the form

$$
\frac{m}{\left(u_{1}, \ldots, u_{n}\right)},
$$

where $m \in M$ and $\left(u_{1}, \ldots, u_{n}\right)=: u \in U$; the above generalized fraction is also written as $m / u$. 
Comparison and addition of generalized fractions involve $n \times n$ lower triangular matrices over (that is with entries in) $R$ : we shall use $L_{n}(R)$ to denote the set of all $n \times n$ lower triangular matrices over $R$, and for $H \in L_{n}(R)$, we shall use $|H|$ to denote the determinant of $H$. Let $x, x^{\prime}, y \in M$ and $u, u^{\prime}, v \in U$. Then $x / u=x^{\prime} / u^{\prime}$ in $U^{-n} M$ if and only if there exist $w=\left(w_{1}, \ldots, w_{n}\right) \in U$ and $H, H^{\prime} \in L_{n}(R)$ such that

$$
H u^{T}=w^{T}=H^{\prime} u^{\prime T} \text { and }|H| x-\left|H^{\prime}\right| x^{\prime} \in \sum_{i=1}^{n-1} w_{i} M .
$$

(We use ${ }^{T}$ to denote matrix transpose, and we use $n$-tuples $\left(a_{1}, \ldots, a_{n}\right)$ of elements of $R$ and $1 \times n$ row matrices $\left(a_{1} \ldots a_{n}\right)$ over $R$ interchangeably.)

The addition and scalar multiplication in $U^{-n} M$ are such that

$$
\frac{x}{u}+\frac{y}{v}=\frac{|H| x+|K| y}{w}
$$

for any choice of $w \in U$ and $H, K \in L_{n}(R)$ such that $H u^{T}=w^{T}=K v^{T}$ (and there is bound to be at least one such choice in view of the definition of triangular set), and

$$
r \frac{x}{u}=\frac{r x}{u}
$$

for $r \in R$. The reader is referred to [23, Section 2] for more details of the construction. Note the following.

2.1 Remark. (See $[23,3.3(i i)]$.) Let $u=\left(u_{1}, \ldots, u_{n}\right) \in U$ (with the above notation). If $m \in \sum_{i=1}^{n-1} u_{i} M$, then $m / u=0$ in $U^{-n} M$.

The next lemma will be needed in Section 3. It shows that, if $U$ consists entirely of poor $M$-sequences, then the converse statement to 2.1 is true. It is a consequence of the Exactness Theorem for generalized fractions, proved in full generality by L. O'Carroll in [14, Theorem 3.1] after Sharp and Zakeri had proved it, in a very complicated manner, in the special case in which the underlying ring is Noetherian in [25, Theorem 3.3]. (We say that $\left(a_{1}, \ldots, a_{n}\right) \in R^{n}$ is a poor $M$-sequence if $a_{i}$ is not a zerodivisor on $M / \sum_{j=1}^{i-1} a_{j} M$ for each $i=1, \ldots, n$; it is an $M$-sequence if, in addition, $M \neq \sum_{j=1}^{n} a_{j} M$.)

2.2 Lemma. Assume that (with the above notation) the triangular subset $U$ of $R^{n}$ consists entirely of poor $M$-sequences. Let $m \in M$ and $u=\left(u_{1}, \ldots, u_{n}\right) \in U$ be such that, in $U^{-n} M$,

$$
\frac{m}{u}=\frac{m}{\left(u_{1}, \ldots, u_{n}\right)}=0
$$

Then $m \in \sum_{i=1}^{n-1} u_{i} M$.

Proof. By the construction of $U^{-n} M$ reviewed at the beginning of this section, there exist $v=\left(v_{1}, \ldots, v_{n}\right) \in U$ and $H=\left(h_{i j}\right) \in L_{n}(R)$ such that

$$
H u^{T}=v^{T} \text { and }|H| m \in \sum_{i=1}^{n-1} v_{i} M .
$$


Let $H^{*}$ denote the top left $(n-1) \times(n-1)$-submatrix of $H$. We now deduce from the above displayed relations, from [14, Theorem 3.2], and from the facts that $H^{*}\left(u_{1}, \ldots, u_{n-1}\right)^{T}=\left(v_{1}, \ldots, v_{n-1}\right)^{T}$ and $\left(v_{1}, \ldots, v_{n-1}\right)$ is a poor $M$-sequence, that

However,

$$
h_{n n} m \in \sum_{i=1}^{n-1} u_{i} M
$$

$$
\left(\begin{array}{c|c}
H^{*} & 0 \\
\hline h_{n, 1} \ldots h_{n, n-1} & u_{n}
\end{array}\right)\left(u_{1}, \ldots, u_{n-1}, h_{n n}\right)^{T}=\left(v_{1}, \ldots, v_{n}\right)^{T},
$$

and so it follows from [14, Theorem 3.2] again that $\left(u_{1}, \ldots, u_{n-1}, h_{n n}\right)$ is a poor $M$-sequence. Hence $m \in \sum_{i=1}^{n-1} u_{i} M$, as claimed.

2.3 Definition and Notation. (See $[14$, p. 420].) By a chain of triangular sets on $R$, we mean a family $U=\left(U_{n}\right)_{n \in \mathbb{N}}$ of sets such that:

(i) $U_{n}$ is a triangular subset of $R^{n}$ for every $n \in \mathbb{N}$;

(ii) (1) $\in U_{1}$;

(iii) for each $n \in \mathbb{N}$ and each $\left(u_{1}, \ldots, u_{n}\right) \in U_{n}$, we have $\left(u_{1}, \ldots, u_{n}, 1\right) \in U_{n+1}$; and

(iv) for each $n \in \mathbb{N}$ with $n>1$, and each $\left(u_{1}, \ldots, u_{n}\right) \in U_{n}$, we have $\left(u_{1}, \ldots, u_{n-1}\right) \in$ $U_{n-1}$.

Such a chain $\mathcal{U}$ determines, for each $R$-module $M$, a complex

$$
0 \longrightarrow M \stackrel{e^{0}}{\longrightarrow} U_{1}^{-1} M \stackrel{e^{1}}{\longrightarrow} \ldots \longrightarrow U_{n}^{-n} M \stackrel{e^{n}}{\longrightarrow} U_{n+1}^{-n-1} M \longrightarrow \ldots
$$

in which $e^{0}(m)=m /(1)$ for all $m \in M$ and

$$
e^{n}\left(\frac{m}{\left(u_{1}, \ldots, u_{n}\right)}\right)=\frac{m}{\left(u_{1}, \ldots, u_{n}, 1\right)}
$$

for all $n \in \mathbb{N}, m \in M$ and $\left(u_{1}, \ldots, u_{n}\right) \in U_{n}$; we shall denote this complex by $C(\mathcal{U}, M)$.

2.4 REMARK. It should be observed that, if $M$ is an $R$-module and $U$ is a triangular subset of $R^{n}$ for some $n \in \mathbb{N}$, then (an isomorphic copy of) the module of generalized fractions $U^{-n} M$ can be incorporated as a term in a complex $C(U, M)$ for a suitable chain $\mathcal{U}$ of triangular sets on $R$. To see this, let $U_{n}$ be the expansion $[23,3.2]$ of $U$, let $U_{m}$ for $m \in \mathbb{N}$ with $m<n$ be the restriction $[23,3.6]$ of $U_{n}$ to $R^{m}$, and, for $q \in \mathbb{N}$ with $q>n$, let

$$
U_{q}=\left\{\left(u_{1}, \ldots, u_{n}, 1, \ldots, 1\right) \in R^{q}:\left(u_{1}, \ldots, u_{n}\right) \in U_{n}\right\}
$$

Then $U=\left(U_{i}\right)_{i \in \mathbb{N}}$ is a chain of triangular sets on $R$, and, by [23, 3.2], there is a natural isomorphism $U^{-n} M \cong U_{n}^{-n} M$.

2.5 Lemma. (See M. H. Bijan-Zadeh [2].) Let $U$ be a triangular subset of $R^{n}$, for some $n \in \mathbb{N}$. Then

$$
\Phi(U):=\left\{\sum_{i=1}^{n} R u_{i}:\left(u_{1}, \ldots, u_{n}\right) \in U\right\}
$$

is a system of ideals of $R$. 
2.6 REMARK. It follows from 2.5 that a chain $U=\left(U_{n}\right)_{n \in \mathbb{N}}$ of triangular sets on $R$ determines a family $\left(\Phi\left(U_{n}\right)\right)_{n \in \mathbb{N}}$ of systems of ideals of $R$, and so the generalized Hughes complex for an $R$-module $M$ with respect to this family can be constructed. It is one of our aims for the next section to show that, in the case when $R$ is Noetherian, this generalized Hughes complex is isomorphic to the complex $C(U, M)$ of modules of generalized fractions of 2.3 .

The next result is a technical lemma which will be of assistance in the next section. Its proof is omitted: it can be proved by routine arguments using generalized fractions, based on [23, 2.2 and 2.3]; alternatively, the reader might like to consider the argument given by Gibson and O'Carroll in [6, 3.3].

2.7 Lemma. (See Gibson and O'Carroll $[6,3.3]$.) Let $M$ be an $R$-module.

(i) Let $U$ be an expanded triangular subset of $R^{1}$. Then $U \times\{1\}$ is a triangular subset of $R^{2}$, and there is an exact sequence

$$
M \stackrel{e}{\longrightarrow} U^{-1} M \stackrel{\omega}{\longrightarrow}(U \times\{1\})^{-2} M \longrightarrow 0,
$$

in which $e$ is the natural homomorphism and $\omega(m /(u))=m /(u, 1)$ for each $m \in M$ and $(u) \in U$.

(ii) Let $U=\left(U_{n}\right)_{n \in \mathbb{N}}$ be a chain of triangular sets on $R$. Choose $n \in \mathbb{N}$. Then $U_{n+1} \times\{1\}$ is a triangular subset of $R^{n+2}$, and there is an exact sequence

$$
U_{n}^{-n} M \stackrel{e^{n}}{\longrightarrow} U_{n+1}^{-n-1} M \stackrel{\omega_{n+1}}{\longrightarrow}\left(U_{n+1} \times\{1\}\right)^{-n-2} M \longrightarrow 0,
$$

in which $e^{n}$ is as defined in 2.3 and $\omega_{n+1}(m / v)=m /(v, 1)$ (with an obvious notation) for each $m \in M$ and $v \in U_{n+1}$.

3. The isomorphisms. In this section we shall work over our commutative Noetherian ring $A$.

3.1 Lemma. (Recall that $A$ is Noetherian.) Let $n \in \mathbb{N}$, let $U$ be an expanded (see [23, 3.2]) triangular subset of $A^{n+1}$ and let $\bar{U}$ be the restriction $[23,3.6]$ of $U$ to $A^{n}$. Let $M$ be an A-module, let $m \in M$ and let $u=\left(u_{1}, \ldots, u_{n+1}\right) \in U$. Note that $\bar{U} \times\{1\}$ is a triangular subset of $A^{n+1}$. Since $A$ is Noetherian, there exists $t \in \mathbb{N}$ such that

$$
\left(A u_{1}+\ldots+A u_{n}: A u_{n+1}^{t}\right)=\left(A u_{1}+\ldots+A u_{n}: A u_{n+1}^{t+1}\right) .
$$

For such data, there exists an A-homomorphism

for which

$$
\delta_{m, u, t}: A u_{1}+\ldots+A u_{n}+A u_{n+1}^{t+1} \rightarrow(\bar{U} \times\{1\})^{-n-1} M
$$

$$
\delta_{m, u, t}\left(\sum_{i=1}^{n} a_{i} u_{i}+a_{n+1} u_{n+1}^{t+1}\right)=\frac{a_{n+1} u_{n+1}^{t} m}{\left(u_{1}, \ldots, u_{n}, 1\right)}
$$

for all $a_{1}, \ldots, a_{n+1} \in A$.

Proof. Let $a_{1}, \ldots, a_{n+1}, b_{1}, \ldots, b_{n+1} \in A$ be such that

$$
\sum_{i=1}^{n} a_{i} u_{i}+a_{n+1} u_{n+1}^{t+1}=\sum_{i=1}^{n} b_{i} u_{i}+b_{n+1} u_{n+1}^{t+1} \text {. }
$$


Then, by choice of $t$,

$$
a_{n+1} u_{n+1}^{t}-b_{n+1} u_{n+1}^{t}=\sum_{i=1}^{n} c_{i} u_{i}
$$

for some $c_{1}, \ldots, c_{n} \in A$, so that, in $(\bar{U} \times\{1\})^{-n-1} M$,

$$
\frac{a_{n+1} u_{n+1}^{t} m}{\left(u_{1}, \ldots, u_{n}, 1\right)}=\frac{b_{n+1} u_{n+1}^{t} m}{\left(u_{1}, \ldots, u_{n}, 1\right)}
$$

in view of 2.1. It follows that there is indeed a mapping $\delta_{m, u, t}$ given by the formula in the statement of the lemma, and it is clear that $\delta_{m, u, i}$ is an $A$-homomorphism.

3.2 Remark. Note that, if, in the situation of 3.1,

$$
\left(A u_{1}+\ldots+A u_{n}: A u_{n+1}^{t}\right)=\left(A u_{1}+\ldots+A u_{n}: A u_{n+1}^{t+1}\right)
$$

then

$$
\left(A u_{1}+\ldots+A u_{n}: A u_{n+1}^{t+k}\right)=\left(A u_{1}+\ldots+A u_{n}: A u_{n+1}^{t+k+1}\right)
$$

for all $k \in \mathbb{N}$.

The next theorem provides the key to the main results of this paper. It establishes a relationship between the modules of generalized fractions of Sharp and Zakeri on the one hand, and the generalized ideal transforms introduced in 1.2 on the other. It should be noted that the Noetherian hypothesis on $A$ is used in an important way.

3.3 Theorem. (Recall that $A$ is Noetherian.) Let the situation be as in 3.1. Thus we let $n \in \mathbb{N}$, we let $U$ be an expanded triangular subset of $A^{n+1}$, we let $\bar{U}$ be the restriction of $U$ to $A^{n}$, and we let $M$ be an A-module. We denote by $\Phi(U)$ the system of ideals of $A$ determined by $U$ (see 2.5 ).

There is an isomorphism of A-modules

$$
\tau_{U}(M): U^{-n-1} M \rightarrow D_{\phi(U)}\left((\bar{U} \times\{1\})^{-n-1} M\right) .
$$

Here $D_{\Phi(U)}$ denotes the $\Phi(U)$-transform (see 1.2). The isomorphism $\tau_{U}(M)$ is described as follows.

For each $\mathfrak{b} \in \Phi(U)$, let

$$
\theta(\Phi(U), \mathfrak{b}): \operatorname{Hom}_{A}\left(\mathfrak{b},(\breve{U} \times\{1\})^{-n-1} M\right) \rightarrow D_{\Phi(U)}\left((\bar{U} \times\{1\})^{-n-1} M\right)
$$

be the canonical homomorphism. For $u=\left(u_{1}, \ldots, u_{n+1}\right) \in U$, it will be convenient to write $\bar{u}=\left(u_{1}, \ldots, u_{n}\right) \in \bar{U}$ and $u=\left(\bar{u}, u_{n+1}\right)$, and to denote $\sum_{i=1}^{n+1} u_{i} A$ by $A u$ and $\sum_{i=1}^{n} u_{i} A$ by $A \bar{u}$. For such $a u$ and for $m \in M$,

$$
\tau_{U}(M)\left(\frac{m}{\left(u_{1}, \ldots, u_{n+1}\right)}\right)=\theta\left(\Phi(U), A \bar{u}+A u_{n+1}^{t+1}\right)\left(\delta_{m, u, t}\right) \in D_{\Phi(U)}\left((\bar{U} \times\{1\})^{-n-1} M\right),
$$

for any $t \in \mathbb{N}$ such that $\left(A \bar{u}: u_{n+1}^{t}\right)=\left(A \bar{u}: u_{n+1}^{t+1}\right)$. (The homomorphism $\delta_{m, u, t}$ was defined in 3.1.) 
Proof. For the purposes of this proof, we shall drop ' $U$ ' and ' $M$ ' from the notation when this can be done without causing confusion. Thus, we shall abbreviate $\tau_{U}(M)$ to $\tau$, $\Phi(U)$ to $\Phi, D_{\Phi(U)}$ to $D_{\Phi}$ and, in the same spirit, we shall abbreviate $\theta(\Phi(U), \mathfrak{b}$ ) (for $\mathfrak{b} \in \Phi(U)=\Phi)$ to $\theta(\mathfrak{b})$.

First note that, for $u \in U$ and $m \in M$ as in the statement of the theorem, it is easy to see that $\theta\left(A \bar{u}+A u_{n+1}^{t+1}\right)\left(\delta_{m, u, t}\right)$ is independent of the choice of $t \in \mathbb{N}$ such that $\left(A \bar{u}: u_{n+1}^{t}\right)=\left(A \bar{u}: u_{n+1}^{t+1}\right)$.

Next, we show that, if $u=\left(u_{1}, \ldots, u_{n+1}\right), v=\left(v_{1}, \ldots, v_{n+1}\right) \in U$ are such that there exists $H=\left(h_{i, j}\right) \in L_{n+1}(A)$ with $H u^{T}=v^{T}$, then, for $m \in M$,

$$
\theta\left(A \bar{u}+A u_{n+1}^{t+1}\right)\left(\delta_{m, u, t}\right)=\theta\left(A \bar{v}+A v_{n+1}^{t+1}\right)\left(\delta_{1 H \mid m, v, t}\right)
$$

for any $t \in \mathbb{N}$ such that

$$
\left(A \bar{u}: u_{n+1}^{t}\right)=\left(A \bar{u}: u_{n+1}^{t+1}\right) \text { and }\left(A \bar{v}: v_{n+1}^{t}\right)=\left(A \bar{v}: v_{n+1}^{t+1}\right) \text {. }
$$

(Of course, there does exist such a $t$.) We shall achieve this by showing that

$$
A \bar{v}+A v_{n+1}^{t+1} \subseteq A \bar{u}+A u_{n+1}^{t+1} \quad \text { and }\left.\quad \delta_{m, u, t}\right|_{A \bar{v}+A u_{n+1}^{t+1}}=\delta_{|H| m, v, t}
$$

To see that this is the case, note first that the equation

$$
v_{n+1}=\sum_{j=1}^{n+1} h_{n+1, j} u_{j}
$$

shows that

$$
v_{n+1}^{t+1}=\sum_{j=1}^{n} d_{j} u_{j}+h_{n+1, n+1}^{t+1} u_{n+1}^{t+1}
$$

for suitable $d_{1}, \ldots, d_{n} \in A$. Hence $A \bar{v}+A v_{n+1}^{t+1} \subseteq A \bar{u}+A u_{n+1}^{t+1}$. Note also that $v_{1}, \ldots, v_{n} \in A \bar{u}$. Let $\bar{H}$ denote the top left $n \times n$ submatrix of $H$, and observe that $m /(\bar{u}, 1)=|\bar{H}| m /(\bar{v}, 1)$ in $(\bar{U} \times\{1\})^{-n-1} M$. It therefore follows that, for all $c_{1}, \ldots, c_{n+1} \in A$, we have

$$
\begin{aligned}
\delta_{m, u, r}\left(\sum_{i=1}^{n} c_{i} v_{i}+c_{n+1} v_{n+1}^{t+1}\right) & =\delta_{m, u, t}\left(c_{n+1} v_{n+1}^{t+1}\right) \\
& =\delta_{m, u, t}\left(c_{n+1}\left(\sum_{j=1}^{n} d_{j} u_{j}+h_{n+1, n+1}^{t+1} u_{n+1}^{t+1}\right)\right) \\
& =\frac{c_{n+1} h_{n+1, n+1}^{t+1} u_{n+1}^{t} m}{(\bar{u}, 1)} .
\end{aligned}
$$

However, we can write $h_{n+1, n+1}^{t} u_{n+1}^{t}=v_{n+1}^{t}+b$ for some $b \in A \bar{u}$, and so it follows from 2.1 that

$$
\begin{aligned}
\delta_{m, u, t}\left(\sum_{i=1}^{n} c_{i} v_{i}+c_{n+1} v_{n+1}^{t+1}\right) & =\frac{c_{n+1} h_{n+1, n+1} v_{n+1}^{t} m}{(\bar{u}, 1)} \\
& =\frac{c_{n+1} h_{n+1, n+1} v_{n+1}^{t}|\bar{H}| m}{(\bar{v}, 1)} \\
& =\delta_{|H| m, v, l}\left(\sum_{i=1}^{n} c_{i} v_{i}+c_{n+1} v_{n+1}^{t+1}\right)
\end{aligned}
$$


because $h_{n+1, n+1}|\bar{H}|=|H|$. Thus

$$
\theta\left(A \bar{u}+A u_{n+1}^{\ell+1}\right)\left(\delta_{m, u, t}\right)=\theta\left(A \bar{v}+A v_{n+1}^{t+1}\right)\left(\delta_{|H| m, v, t}\right),
$$

as claimed.

It is now an easy matter to deduce from the last three paragraphs that there is indeed a mapping

$$
\tau=\tau_{U}(M): U^{-n-1} M \rightarrow D_{\Phi}\left((\bar{U} \times\{1\})^{-n-1} M\right)
$$

given by the formula in the statement of the theorem. It is routine to check that $\tau$ is an $A$-homomorphism. It only remains for us to show that $\tau$ is bijective.

Let $m \in M$ and $u \in U$ be such that $m / u \in \operatorname{Ker} \tau$. Let $t \in \mathbb{N}$ be such that $\left(A \bar{u}: u_{n+1}^{t}\right)=$ $\left(A \bar{u}: u_{n+1}^{t+1}\right)$. Then there exists $v \in U$ such that

$$
A v \subseteq A \bar{u}+A u_{n+1}^{t+1} \quad \text { and }\left.\quad \delta_{m, u, t}\right|_{A v}=0 .
$$

Since $U$ is a triangular subset of $A^{n+1}$, there exist $w \in U$ and $H, K \in L_{n+1}(A)$ such that $H u^{T}=w^{T}=K v^{T}$. Choose $t^{\prime} \in \mathbb{N}$ such that $t \leqslant t^{\prime}$ and

$$
\left(A \bar{w}: w_{n+1}^{t^{\prime}}\right)=\left(A \bar{w}: w_{n+1}^{t^{\prime}+1}\right) .
$$

By 3.2, $\left(A \bar{u}: u_{n+1}^{t^{\prime}}\right)=\left(A \bar{u}: u_{n+1}^{t^{\prime}+1}\right)$. Let $\bar{H}$ denote the top left $n \times n$ submatrix of $H$. It follows from the paragraph before last in this proof that

$$
\delta_{|H| m, w, t^{\prime}}=\left.\left(\delta_{m, u, t^{\prime}}\right)\right|_{A \bar{w}+A w_{n+1}^{t^{\prime \prime}+1}}=0 .
$$

In particular, $\delta_{|H| m, w, r^{\prime}}\left(w_{n+1}^{t^{\prime}+1}\right)=0$, so that

$$
\frac{w_{n+1}^{c^{\prime}}|H| m}{\left(w_{1}, \ldots, w_{n}, 1\right)}=0
$$

in $(\bar{U} \times\{1\})^{-n-1} M$. Since $U$ is expanded, it follows easily from this that

$$
\frac{w_{n+1}^{t^{\prime}+1}|H| m}{\left(w_{1}, \ldots, w_{n+1}\right)}=\frac{w_{n+1}^{t^{\prime}}|H| m}{\left(w_{1}, \ldots, w_{n}, 1\right)}=0
$$

in $U^{-n-1} M$. Now use $[24,2.1]$ to see that $m / u=|H| m / w=0$. Thus $\tau$ is injective.

Finally, we show that $\tau$ is surjective. Let $\alpha \in D_{\Phi}\left((\bar{U} \times\{1\})^{-n-1} M\right)$; thus there exists $\mathfrak{b} \in \Phi$, say $\mathfrak{b}=\sum_{i=1}^{n+1} A u_{i}$, where $u=\left(u_{1}, \ldots, u_{n+1}\right) \in U$, and

$$
\beta \in \operatorname{Hom}_{A}\left(\mathfrak{b},(\bar{U} \times\{1\})^{-n-1} M\right)
$$

such that $\theta(\mathfrak{b})(\beta)=\alpha$.

Since $\mathfrak{b}$ is finitely generated and finitely many generalized fractions in

$$
(\bar{U} \times\{1\})^{-n-1} M
$$

can be put on a common denominator, there exists $v \in U$ such that

$$
\operatorname{Im} \beta \subseteq\left\{\frac{z}{(\bar{v}, 1)}: z \in M\right\},
$$

a submodule of $(\bar{U} \times\{1\})^{-n-1} M$. Furthermore, by use of the properties of triangular sets, we can in addition arrange that $A v \subseteq A u=\mathfrak{b}$. Note that $\beta\left(v_{i}^{2}\right)=0$ for all $i=1, \ldots, n$, by 
2.1. Set

$$
\beta\left(v_{n+1}\right)=\frac{m}{(\bar{v}, 1)}=\frac{v_{1} \ldots v_{n} m}{\left(v_{1}^{2}, \ldots, v_{n}^{2}, 1\right)}
$$

for an appropriate $m \in M$. Now choose $t \in \mathbb{N}$ such that

$$
\left(\sum_{i=1}^{n} A v_{i}^{2}: v_{n+1}^{t}\right)=\left(\sum_{i=1}^{n} A v_{i}^{2}: v_{n+1}^{t+1}\right)
$$

and observe that

$$
\left.\beta\right|_{\sum_{i=1}^{n} A v_{i}^{2}+A v_{n+1}^{c+1}}=\delta_{v_{1} \ldots v_{n} m,\left(v_{1}^{2}, \ldots, v_{n}^{2}, v_{n+1}\right), t}
$$

and

$$
\alpha=\theta(\mathfrak{b})(\beta)=\theta\left(\sum_{i=1}^{n} A v_{i}^{2}+A v_{n+1}^{\ell+1}\right)\left(\delta_{v_{1} \ldots v_{n} m,\left(v_{1}^{2}, \ldots, v_{n}^{2}, v_{n+1}\right), \ell}\right) \in \operatorname{Im} \tau
$$

in order to complete the proof.

A similar result is available for triangular subsets of $A^{1}$. As this is reminiscent of work with ordinary modules of fractions, and as its proof is similar to, but simpler than, the above proof of 3.3, we merely state the result here and leave the proof to the reader.

3.4 THEOREM. (Recall that $A$ is Noetherian.) Let $U$ be an expanded triangular subset of $A^{1}$, and let $M$ be an A-module. We denote by $\Phi(U)$ the system of ideals of A determined by $U$ (see 2.5).

There is an isomorphism of $A$-modules

$$
\tau_{U}(M): U^{-1} M \rightarrow D_{\Phi(U)}(M) .
$$

Here $D_{\Phi(U)}$ denotes the $\Phi(U)$-transform (see 1.2). The isomorphism $\tau_{U}(M)$ is described as follows.

For each $\mathfrak{b} \in \Phi(U)$, let

$$
\theta(\Phi(U), \mathfrak{b}): \operatorname{Hom}_{A}(\mathfrak{b}, M) \rightarrow D_{\Phi(U)}(M)
$$

be the canonical homomorphism. For $(u) \in U$ and $m \in M$,

$$
\tau_{U}(M)(m /(u))=\theta\left(\Phi(U), A u^{t+1}\right)\left(\varepsilon_{m, u, t}\right)
$$

where $t \in \mathbb{N}$ is chosen such that $\left(0: u^{t}\right)=\left(0: u^{t+1}\right)$ and $\varepsilon_{m, u, t}: A u^{t+1} \rightarrow M$ is such that $\varepsilon_{m, u, t}\left(a u^{t+1}\right)=a u^{t} m$ for all $a \in A$.

Next, we use Theorems 3.3 and 3.4 to show that, for a chain $U=\left(U_{n}\right)_{n \in \mathbb{N}}$ of triangular sets on $A$ and an $A$-module $M$, the induced complex $C(U, M)$ of modules of generalized fractions

$$
0 \longrightarrow M \stackrel{e^{0}}{\longrightarrow} U_{1}^{-1} M \stackrel{e^{1}}{\longrightarrow} \ldots \longrightarrow U_{n}^{-n} M \stackrel{e^{n}}{\longrightarrow} U_{n+1}^{-n-1} M \longrightarrow \ldots
$$

is isomorphic to the generalized Hughes complex for $M$ with respect to the family of systems of ideals determined by $U$ (see 2.6).

3.5 TheOREM. (Recall that $A$ is Noetherian.) Let $U=\left(U_{n}\right)_{n \in \mathbb{N}}$ be a chain of triangular sets on $A$, and let $M$ be an $A$-module. Denote the complex $C(U, M)$ of modules of 
generalized fractions by

$$
0 \longrightarrow M \stackrel{f^{-1}}{\longrightarrow} F^{0} \stackrel{f^{0}}{\longrightarrow} F^{1} \longrightarrow \ldots \longrightarrow F^{n} \stackrel{f^{n}}{\longrightarrow} F^{n+1} \longrightarrow \ldots
$$

(so that $F^{n}=U_{n+1}^{-n-1} M$ and $f^{n-1}=e^{n}$ for all $n \in \mathbb{N}_{0}$ ), and set $F^{-1}=M$.

Let $\mathscr{S}(U)=\left(\Phi\left(U_{n}\right)\right)_{n \in \mathbb{N}}$ be the family of systems of ideals of $A$ determined by $U$ (see 2.6). Denote the generalized Hughes complex (see 1.3) $\mathscr{H}(\mathscr{S}(\mathscr{U}), M)$ for $M$ with respect to $\mathscr{P}(\mathcal{Q})$ by

$$
0 \longrightarrow M \stackrel{h^{-1}}{\longrightarrow} K^{0} \stackrel{h^{0}}{\longrightarrow} K^{1} \longrightarrow \ldots \longrightarrow K^{n} \stackrel{h^{n}}{\longrightarrow} K^{n+1} \longrightarrow \ldots,
$$

and set $K^{-1}=M$.

Then there is an isomorphism of complexes

$$
\Psi=\left(\psi^{i}\right)_{i \geq-2}: C(\mathcal{U}, M) \rightarrow \mathscr{H}(\mathscr{P}(\mathcal{U}), M)
$$

such that $\psi^{-1}: F^{-1} \rightarrow K^{-1}$ is the identity mapping on $M$. Moreover, for each $n \in \mathbb{N}_{0}$,

$$
\text { Coker } f^{n-1} \cong \text { Coker } h^{n-1} \cong\left(U_{n+1} \times\{1\}\right)^{-n-2} M \text {. }
$$

Proof. The isomorphism $\Psi=\left(\psi^{i}\right)_{i \geqslant-2}$ is constructed by a straightforward inductive process, and the details are left to the reader. Use 3.4 to define $\psi^{0}$, and use 2.7 and 3.3 for the inductive step.

3.6 Remark. It is now immediate from 2.4 and 3.5 that, if $M$ is an arbitrary module over our commutative Noetherian ring $A$, and $U$ is an arbitrary triangular subset of $A^{n}$ for some $n \in \mathbb{N}$, then the module of generalized fractions $U^{-n} M$ is isomorphic to the $(n-1)$-st term in an appropriate generalized Hughes complex for $M$. We would argue that this fact adds to the usefulness of the generalized fractions of Sharp and Zakeri (as opposed to making them redundant in Noetherian situations), because the module $U^{-n} M$ is constructed in one step, and calculations with its elements are a little like calculations in ordinary modules of fractions, whereas the $(n-1)$-st term in a generalized Hughes complex for $M$ is arrived at after $n$ direct limit constructions.

3.7 Remark. In support of the comment made in 3.6 above, let us reconsider Hughes' grade-theoretic analogue of the Cousin complex. By 1.4, this is just the generalized Hughes complex $\mathscr{H}(\mathscr{G}, A)$ of $A$ with respect to $\mathscr{G}$, the family of systems of ideals of our commutative Noetherian ring $A$ given by $\mathscr{G}=(\mathscr{G}(n))_{n \in \mathbb{N}}$, where, for each $n \in \mathbb{N}$, the system $\mathscr{G}(n)$ is the set of all ideals of $A$ of grade at least $n$.

However, we can give another description of this complex. For each $n \in \mathbb{N}$, let

$$
V_{n}=\left\{\left(v_{1}, \ldots, v_{n}\right) \in A^{n}:\left(v_{1}, \ldots, v_{n}\right) \text { is a poor } A \text {-sequence }\right\} \text {. }
$$

By [23, 3.9 and 3.10], $V_{n}$ is a triangular subset of $A^{n}$, and, in fact, it is easy to see that $\mathscr{V}:=\left(V_{n}\right)_{n \in \mathbb{N}}$ is a chain of triangular sets on $A$. Therefore, by 3.5 , there is an isomorphism of complexes

$$
C(\mathscr{V}, A) \stackrel{\cong}{\longrightarrow} \mathscr{H}(\mathscr{P}(\mathscr{V}), A),
$$

from the complex $C(\mathscr{V}, A)$ of modules of generalized fractions to the generalized Hughes complex $\mathscr{H}(\mathscr{S}(\mathscr{V}), A)$ for $A$ with respect to the family $\mathscr{S}(\mathscr{V})=\left(\Phi\left(V_{n}\right)\right)_{n \in \mathbb{N}}$ of systems of ideals of $A$ determined by $\mathscr{V}$. However, for each $n \in \mathbb{N}$, the system $\Phi\left(V_{n}\right)$ is just the set of 
all ideals of $A$ which can be generated by a poor $A$-sequence of length $n$, and so is a cofinal subset of $\mathscr{G}(n)$ (partially ordered by reverse inclusion). It is therefore easy to see that Hughes' complex $\mathscr{H}(\mathscr{G}, A)$ is isomorphic to the complex $\mathscr{H}(\mathscr{Y}(\mathscr{V}), A)$, and therefore to $C(\mathscr{V}, A)$.

We next use the above ideas to obtain quickly some properties of the complex $\mathscr{H}(\mathscr{G}, A)$ constructed by Hughes. That listed in part (iii) of Corollary 3.8 below was actually brought by Hughes into his construction in [10] (although with little indication of proof); however, as we showed in 1.3 and 1.4, one does not need to use this property in order to construct $\mathscr{H}(\mathscr{G}, A)$.

3.8 Corollary. (Recall that $A$ is Noetherian.) Denote Hughes' grade-theoretic analogue of the Cousin complex $\mathscr{H}(\mathcal{G}, A)$ by

$$
0 \stackrel{f^{-2}}{\longrightarrow} A \stackrel{f^{-1}}{\longrightarrow} D^{0} \stackrel{f^{0}}{\longrightarrow} D^{1} \longrightarrow \ldots \longrightarrow D^{n} \stackrel{f^{n}}{\longrightarrow} D^{n+1} \longrightarrow \ldots,
$$

and recall (from the Introduction or from 1.3) the A-modules $E^{n}\left(n \in \mathbb{N}_{0}\right)$ used in its construction: they are such that $E^{n} \cong \operatorname{Coker} f^{n-2}$ for all $n \in \mathbb{N}_{0}$. Then

(i) $\mathscr{H}(\mathscr{G}, A)$ is exact,

(ii) for each $n \in \mathbb{N}_{0}$, each non-zero element of $D^{n}$ has annihilator of grade exactly $n$, and

(iii) for each $n \in \mathbb{N}_{0}$, each non-zero element of $E^{n}$ has annihilator of grade exactly $n$.

Proof. By $3.7, \mathscr{H}(\mathscr{G}, A) \cong C(\mathscr{V}, A)$, where $\mathscr{V}:=\left(V_{n}\right)_{n \in \mathbb{N}}$ is the chain of triangular sets on $A$ given by

$$
V_{n}=\left\{\left(v_{1}, \ldots, v_{n}\right) \in A^{n}:\left(v_{1}, \ldots, v_{n}\right) \text { is a poor } A \text {-sequence }\right\}
$$

for each $n \in \mathbb{N}$. Instead of working with $\mathscr{H}(\mathscr{G}, A)$, we work with $C(\mathscr{V}, A)$, which has the form

$$
0 \longrightarrow A \stackrel{e^{0}}{\longrightarrow} V_{1}^{-1} A \stackrel{e^{1}}{\longrightarrow} \ldots \longrightarrow V_{n}^{-n} A \stackrel{e^{n}}{\longrightarrow} V_{n+1}^{-n-1} A \longrightarrow \ldots
$$

(i) It is immediate from the Exactness Theorem for generalized fractions (see [14, Theorem 3.1] or [25, Theorem 3.3]) that $C(\mathscr{V}, A)$ is exact.

(ii) Let $n \in \mathbb{N}_{0}$. By 3.7, $D^{n} \cong V_{n+1}^{-n-1} A$. A non-zero element $\alpha$ of this must have the form $a /\left(v_{1}, \ldots, v_{n}, v_{n+1}\right)$ for some $a \in A$ and $\left(v_{1}, \ldots, v_{n}, v_{n+1}\right) \in V_{n+1}$. By 2.1, we must have $\sum_{i=1}^{n} A v_{i} \neq A$, and, by 2.2 ,

$$
(0: \alpha)=\left(A v_{1}+\ldots+A v_{n}: a\right) .
$$

It is easy to deduce from the theory of grade that the latter ideal has grade exactly $n$.

(iii) Let $n \in \mathbb{N}_{0}$. Since $E^{n} \cong$ Coker $f^{n-2}$, it follows from part (i) that $E^{n}$ is isomorphic to a submodule of $D^{n}$, and so the result follows immediately from part (ii).

3.9 Remark. Now let $M$ be a module over our commutative Noetherian ring $A$ with the property that $\operatorname{Ass}(M)$ contains only finitely many minimal members. (This latter condition is, of course, satisfied if $M$ is finitely generated.) Let $\mathscr{F}$ be a filtration of $\operatorname{Spec}(A)$ which admits $M([21,1.1])$. Then it follows from $[18,(3.4)]$ that there is a chain $\mathcal{U}$ of triangular sets on $A$ such that the Cousin complex $C(\mathscr{F}, M)$ for $M$ with respect to $\mathscr{F}$ is isomorphic to the complex $C(U, M)$ of modules of generalized fractions. Hence, by 
3.5 , the Cousin complex $C(\mathscr{F}, M)$ is isomorphic to the generalized Hughes complex $\mathscr{H}(\mathscr{Y}(U), M)$ for $M$. Hence 'grade-theoretic analogue of the Cousin complex' is indeed an appropriate name for the complex constructed by Hughes in [10].

Let us apply the above ideas to the Cousin complex $C(A)$ for $A$ mentioned in the first few lines of this paper. In this case, $U:=\left(U_{n}\right)_{n \in \mathbb{N}}$ is such that, for each $n \in \mathbb{N}$,

$$
U_{n}=\left\{\left(u_{1}, \ldots, u_{n}\right) \in A^{n}: \operatorname{ht}\left(A u_{1}+\ldots+A u_{i}\right) \geqslant i \text { for all } i=1, \ldots, n\right\} .
$$

(We interpret the height of the improper ideal $A$ of $A$ as $\infty$.) Thus $\mathscr{S}(\mathscr{U})=\left(\Phi\left(U_{n}\right)\right)_{n \in \mathbb{N}}$ is such that, for each $n \in \mathbb{N}$,

$$
\Phi\left(U_{n}\right)=\left\{\sum_{j=1}^{n} A u_{j}:\left(u_{1}, \ldots, u_{n}\right) \in A^{n} \text { and ht }\left(\sum_{j=1}^{i} A u_{j}\right) \geqslant i(1 \leqslant i \leqslant n)\right\} .
$$

We can give another description of $\mathscr{H}(\mathscr{P}(\mathcal{U}), A)$. For each $n \in \mathbb{N}$, set

$$
\mathscr{R}(n)=\{\mathfrak{b}: \mathfrak{b} \text { is an ideal of } A \text { and } h \mathrm{~b} \mathfrak{b} \geqslant n\} .
$$

For each $n \in \mathbb{N}$, the set $\mathscr{R}(n)$ is a system of ideals of $A$, and one can see by prime avoidance arguments that $\Phi\left(U_{n}\right)$ is a cofinal subset of $\mathscr{R}(n)$ (partially ordered by reverse inclusion). Let $\mathscr{R}$ denote the family of systems of ideals $(\mathscr{R}(n))_{n \in \mathbb{N}}$. It follows that we have isomorphisms of complexes

$$
C(A) \cong C(\mathscr{U}, A) \cong \mathscr{H}(\mathscr{S}(\mathcal{U}), A) \cong \mathscr{H}(\mathscr{R}, A) .
$$

Of course, when $A$ is Cohen-Macaulay, $\mathscr{R}=\mathscr{G}$ and Hughes' complex $\mathscr{H}(\mathscr{G}, A)$ is then $\mathscr{L}(\mathscr{R}, A)$ and so is isomorphic to $C(A)$. Thus, in view of $[19,(5.4)]$, we recover the fact stated by Hughes in [10] that his complex provides the minimal injective resolution for $A$ in the case when $A$ is a Gorenstein ring.

It should be noted that we have made considerable use in this section of the Noetherian property of $A$. Indeed, it is perhaps interesting to ask whether there is any analogue of Theorem 3.5 for our commutative ring $R$ which is not necessarily Noetherian. Up to the time of writing, we have not found a complete answer to this question.

\section{REFERENCES}

1. M. H. Bijan-Zadeh, A common generalization of local cohomology theories, Glasgow Math. J. 21 (1980), 173-181.

2. M. H. Bijan-Zadeh, Modules of generalized fractions and general local cohomology modules, Arch. Math. (Basel) 48 (1987), 58-62.

3. D. Flores de Chela, Flat dimensions of certain modules of generalized fractions, Quart $J$. Math. Oxford Ser. (2) 36 (1985), 413-423.

4. G. J. Gibson, Modules of generalized fractions, matrices and determinantal maps, $J$. London Math. Soc. (2) 33 (1986), 245-252.

5. G. J. Gibson, Direct limit systems and flat dimension of generalized fractions, Quart. J. Math. Oxford Ser. (2) 38 (1987), 313-319.

6. G. J. Gibson and L. O'Carroll, Direct limit systems, generalized fractions and complexes of Cousin type, J. Pure Appl. Algebra 54 (1988), 249-259.

7. A. Grothendieck, Local cohomology, Lecture Notes in Mathematics 41 (Springer, 1967).

8. M. A. Hamieh and R. Y. Sharp, Krull dimension and generalized fractions, Proc. Edinburgh Math. Soc. (2) 28 (1985), 349-353.

9. M. A. Hamieh and H. Zakeri, Denominator systems and modules of generalized fractions, J. London Math. Soc. (2) 33 (1986), 237-244. 
10. K. R. Hughes, A grade-theoretic analogue of the Cousin complex, Quaestiones Math. 9 (1986), 293-300.

11. J. Lambek, Torsion theories, additive semantics, and rings of quotients, Lecture Notes in Mathematics 177 (Springer, 1971).

12. I. G. Macdonald and R. Y. Sharp, An elementary proof of the non-vanishing of certain local cohomology modules, Quart. J. Math. Oxford Ser. (2) 23 (1972), 197-204.

13. H. Matsumura, Commutative ring theory (Cambridge University Press, 1986).

14. L. O'Carroll, On the generalized fractions of Sharp and Zakeri, J. London Math. Soc. (2) 28 (1983), 417-427.

15. L. O'Carroll, Generalized fractions, determinantal maps, and top cohomology modules, J. Pure Appl. Algebra 32 (1984), 59-70. 1960).

16. D. G. Northcott, An introduction to homological algebra (Cambridge University Press,

17. D. Rees, The grade of an ideal or module, Proc. Cambridge Philos. Soc. 53 (1957), 28-42.

18. A. M. Riley, R. Y. Sharp and H. Zakeri, Cousin complexes and generalized fractions, Glasgow Math. J. 26 (1985), 51-67.

19. R. Y. Sharp, The Cousin complex for a module over a commutative Noetherian ring, Math. Z. 112 (1969), 340-356.

20. R. Y. Sharp, Local cohomology theory in commutative algebra, Quart. J. Math. Oxford Ser. (2) 21 (1970), 425-434.

21. R. Y. Sharp, A Cousin complex characterization of balanced big Cohen-Macaulay modules, Quart. J. Math. Oxford Ser. (2) 33 (1982), 471-485.

22. R. Y. Sharp and M. A. Hamieh, Lengths of certain generalized fractions, J. Pure Appl. Algebra 38 (1985), 323-336. $32-41$.

23. R. Y. Sharp and H. Zakeri, Modules of generalized fractions, Mathematika 29 (1982),

24. R. Y. Sharp and H. Zakeri, Local cohomology and modules of generalized fractions, Mathematika 29 (1982), 296-306.

25. R. Y. Sharp and H. Zakeri, Modules of generalized fractions and balanced big Cohen-Macaulay modules, Commutative algebra: Durham 1981, London Mathematical Society Lecture Notes 72 (Cambridge University Press, 1982), 61-82.

26. R. Y. Sharp and $\mathrm{H}$. Zakeri, Guneralized fractions and the monomial conjecture, $J$. Algebra 92 (1985), 380-388.

27. R. Y. Sharp and H. Zakeri, Generalized fractions, Buchsbaum modules, and generalized Cohen-Macaulay modules, Math. Proc. Cambridge Philos. Soc. 98 (1985), 429-436.

28. H. Zakeri, d-sequences, local cohomology modules and generalized analytic independence, Mathematika 33 (1986), 279-284.

Department of Pure Mathematics

UNIVERSITY OF SHEFFIELD

Hicks BUILDING

SHEFFIELD S3 7RH 